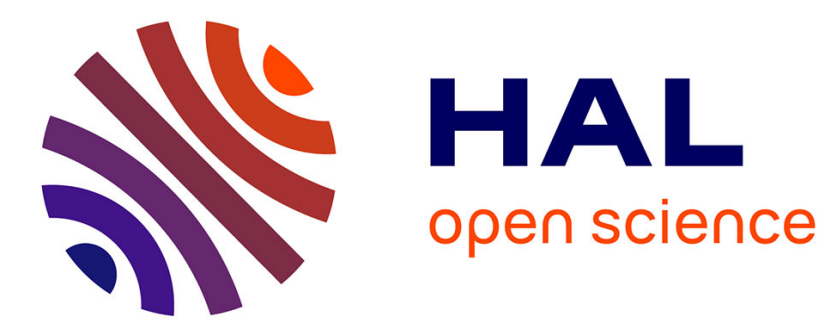

\title{
Lezha [Lissos, Alessio] (Albanie)
}

\author{
Luc Buchet, Etleva Nallbani, Surja Lela
}

\section{To cite this version:}

Luc Buchet, Etleva Nallbani, Surja Lela. Lezha [Lissos, Alessio] (Albanie) : Fouilles d'urgence dans l'espace funéraire (2011). Chronique des activités archéologiques de l'École française de Rome, 2012, 10.4000/cefr.684 . halshs-01793373

\section{HAL Id: halshs-01793373 https://shs.hal.science/halshs-01793373}

Submitted on 16 May 2018

HAL is a multi-disciplinary open access archive for the deposit and dissemination of scientific research documents, whether they are published or not. The documents may come from teaching and research institutions in France or abroad, or from public or private research centers.
L'archive ouverte pluridisciplinaire HAL, est destinée au dépôt et à la diffusion de documents scientifiques de niveau recherche, publiés ou non, émanant des établissements d'enseignement et de recherche français ou étrangers, des laboratoires publics ou privés. 


\section{Lezha [Lissos, Alessio] (Albanie)}

Fouilles d'urgence dans l'espace funéraire (2011)

\section{Luc Buchet, Etleva Nallbani et Surja Lela}

École française de Rome, Institut archéologique d'Albanie, Centre d'histoire et civilisation de Byzance (UMR 8167 - « Orient et Méditerranée », Paris), CEPAM (UMR 6130 UNS-CNRS Nice), INED (Paris), Direction de l'archéologie préventive de la Communauté d'agglomération de Douaisis (DARCAD), Service d'archéologie préventive d'Albanie (Ministère du Tourisme et de la Culture), Parc archéologique de Lezha et Municipalité de Lezha (éd.)

\section{revues.org}

\section{Éditeur}

École française de Rome

Édition électronique

URL : http://cefr.revues.org/684

DOI : $10.4000 /$ cefr.684

ISSN : 2282-5703

Référence électronique

Luc Buchet, Etleva Nallbani et Surja Lela, «Lezha [Lissos, Alessio] (Albanie) », Chronique des activités archéologiques de l'École française de Rome [En ligne], Balkans, mis en ligne le 19 décembre 2012, consulté le 01 octobre 2016. URL : http://cefr.revues.org/684 ; DOI : 10.4000/cefr.684

Ce document a été généré automatiquement le 1 octobre 2016.

(c) École française de Rome 


\title{
Lezha [Lissos, Alessio] (Albanie)
}

\author{
Fouilles d'urgence dans l'espace funéraire (2011)
}

\author{
Luc Buchet, Etleva Nallbani et Surja Lela
}

École française de Rome, Institut archéologique d'Albanie, Centre d'histoire et civilisation de Byzance (UMR 8167 - « Orient et Méditerranée », Paris), CEPAM (UMR 6130 UNS-CNRS Nice), INED (Paris), Direction de l'archéologie préventive de la Communauté d'agglomération de Douaisis (DARCAD), Service d'archéologie préventive d'Albanie (Ministère du Tourisme et de la Culture), Parc archéologique de Lezha et Municipalité de Lezha (éd.)

\section{NOTE DE L'ÉDITEUR}

Le programme est co-dirigé par L. Buchet (anthropologue et archéologue au CEPAM/UMR 6130, Nice et à l'INED) et E. Nallbani (archéologue à l'École française de Rome et au CHCB/ UMR 8167, Paris). L'équipe était composée de S. Lela, M. Meshini et S. Bushi (archéologues au Service de l'archéologie préventive du Ministère du Tourisme et de la Culture albanais, AshA), B. Bregu (étudiante à l'Université de Tirana), E. Metalla (chercheur à l'Institut archéologique d'Albanie à Tirana), H. Sokoli (archéologue au Parc archéologique de Shkodra) ; M. Buchet (anthropologue contractuelle au CEPAM, Nice), G. Dehais (doctorante à l'Université de Nice-Sophia Antipolis), S. Vattéoni (archéologue à la Direction de l'archéologie préventive de la Communauté d'agglomération du Douaisis, Douai), G. Wilson (étudiant à l'Université de Nice-Sophia Antipolis). Les travaux de débroussaillage et de terrassement ont été assurés par deux pelles mécaniques et quinze ouvriers de Lezha. La restauration des objets est assurée par E. Bitri (restaurateur au Studio de restauration et conservation de l'Art, Tirana). Qu'ils soient ici tous remerciés. Le projet, planifié pour cinq ans, est soutenu et financé, pour l'Albanie, par l'Institut archéologique d'Albanie du Centre des études albanologiques (Tirana) et Albanian Development Fund, pour cette dernière campagne de fouilles, avec l'aide ponctuelle de la Municipalité de Lezha ; pour la France, par l'École française de Rome, le Centre d'études Préhistoire, Antiquité, Moyen Âge (UMR 6130, UNS-CNRS, Valbonne), l'Institut national d'études démographiques (Paris) et le Centre d'histoire et civilisation de Byzance (UMR 8167 - Orient et Méditerranée, Paris). 
1 En 2011, la mission franco-albanaise de coopération archéologique à Lezha (Albanie) ${ }^{1}$ a mené deux opérations : une mission d'étude, du 16 au 30 avril et une campagne de fouilles du 5 août au 15 septembre.

1. La mission de printemps a été consacrée :

- à l'achèvement du lavage des ossements exhumés lors de la dernière campagne ;

- à la poursuite de l'étude anthropologique ;

- à la photographie, au dessin et à l'étude du matériel ;

- à la mise au propre des relevés (DAO).

2. Les fouilles de la campagne d'été ont porté exclusivement sur la zone menacée par la construction d'une route qui doit remplacer le chemin actuel menant à la citadelle ; l'achèvement des travaux est prévu pour la fin de l'année 2011. Le tracé de cette route, qui traverse d'est en ouest l'espace funéraire, conduisait inévitablement à détruire des structures qui n'avaient pas encore fait l'objet de fouilles. Ce tracé a été surveillé et ensuite fouillé sur une longueur de $230 \mathrm{~m}$ environ et une largeur de $6 \mathrm{~m}$, ainsi que l'emplacement d'un futur parking au sud-est de la route $(20 \times 30 \mathrm{~m})$.

2 Les apports majeurs de cette campagne peuvent se résumer en trois points: un accroissement très important du nombre de tombes fouillées et, par voie de conséquence, une augmentation significative de l'échantillon archéo-anthropologique ; la mise au jour d'une chapelle, située à $15 \mathrm{~m}$ environ à l'est de l'église de la zone 1 ; le dégagement complet d'une citerne accolée à cette église.

\section{Le cimetière (zones $1,2,3,7$ et 8 )}

3 Quatre-vingt-douze nouvelles tombes ont été repérées, ce qui porte à 178 le nombre total de sépultures identifiées. Elles se répartissent dans les zones 3 (la plus à l'ouest du cimetière), 1 et 2 (près de l'église), 7 (près de la chapelle) et 8 (la plus à l'est).

Dans ce nouvel ensemble, une tombe bâtie et enduite de mortier ne contenait aucun vestige osseux; de même, deux fosses, taillées dans la roche, étaient également vides d'ossements et comblées de pierres, ce qui rend leur fonction funéraire douteuse ; treize sépultures n'ont pas été fouillées car elles ne se trouvaient plus directement menacées par la construction de la route grâce à la possibilité que nous avons obtenue de modifier le tracé.

5 Cela porte donc à soixante-seize le nombre de tombes fouillées pendant la campagne de 2011.

6 Les fouilles des années précédentes avaient permis d'élaborer une première typologie des tombes ; chacun de ces types a été retrouvé cette année.

7 Les tombes les plus nombreuses (25) sont celles qui se présentent sous la forme d'un coffre de dalles (fig. 1 et 2). 
Fig. 1 - Lezha. Tombe 150 (coffre de dalles).

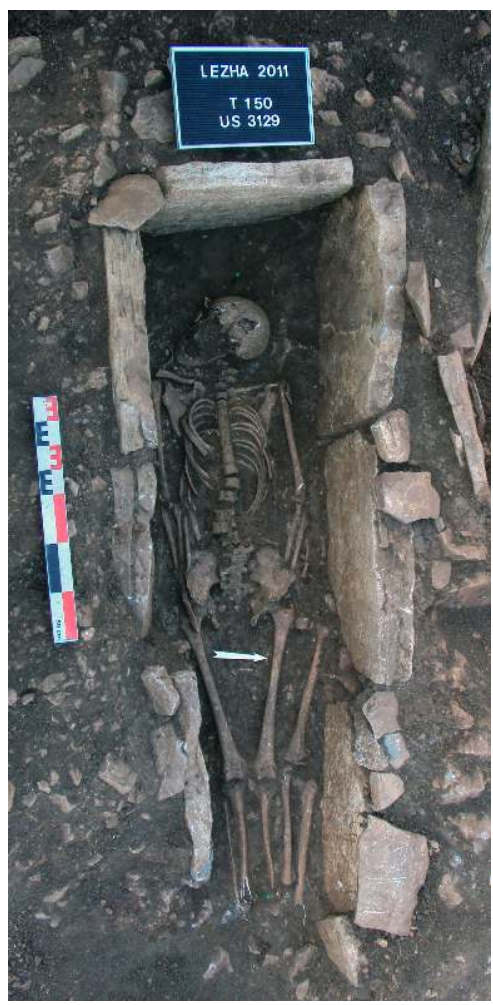

Fig. 2 - Lezha. Tombe 150 (couverture en dalles).

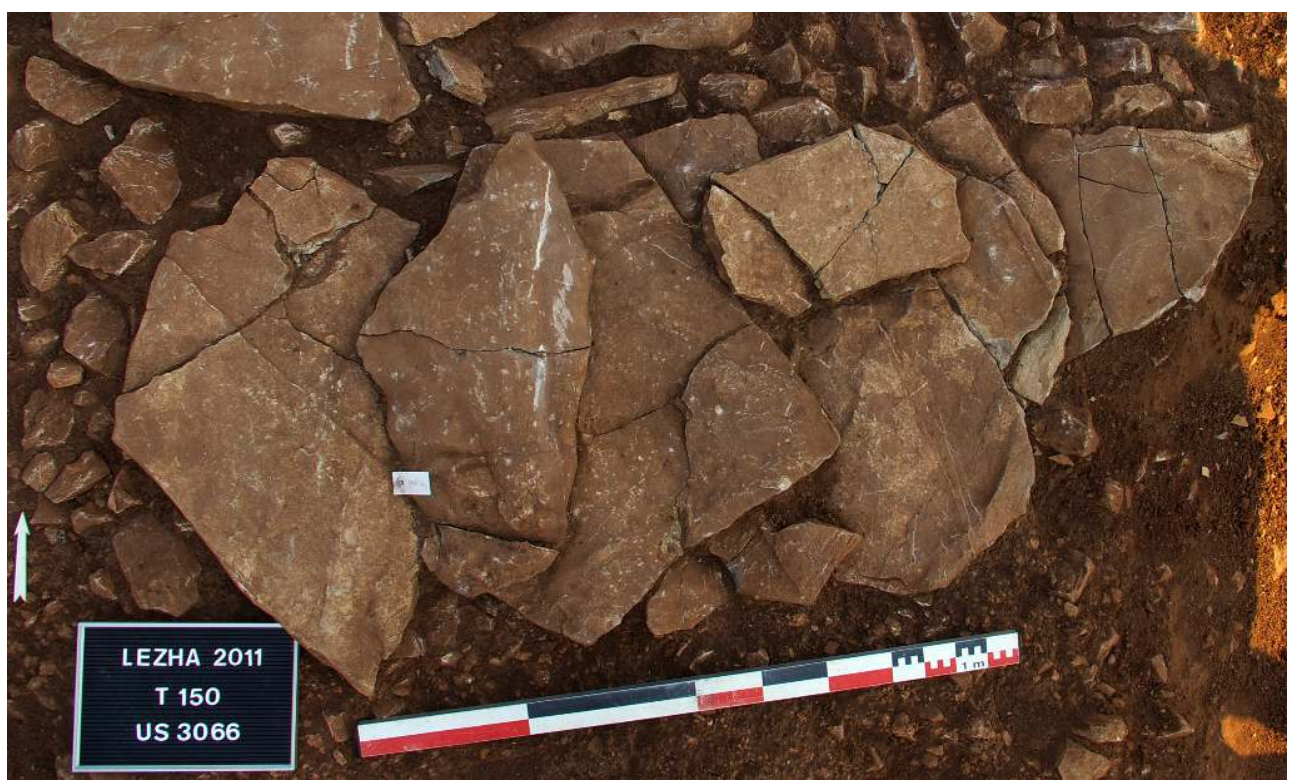

8 Viennent ensuite les tombes dites en pleine terre car aucun élément de structure contenant le corps n'a pu être identifié (17) (fig. 3). 
Fig. 3 - Lezha. Tombe 138.

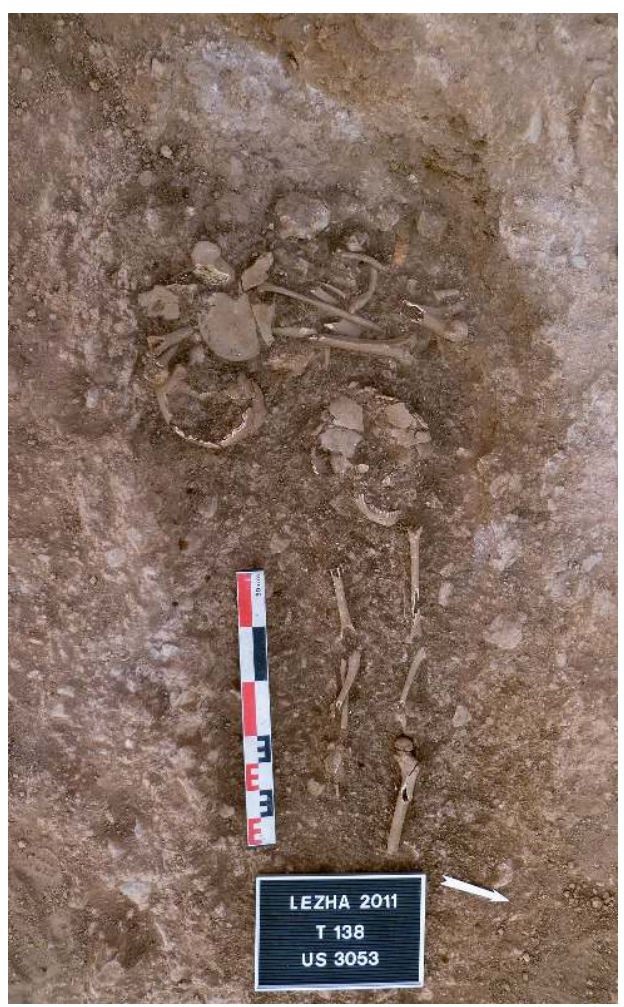

9 Dans ce cas, les fosses, taillées dans la roche, sont peu profondes. Presqu'aussi nombreuses, les tombes mixtes - parois en moellons et couverture de dalles - (15) (fig. 4 et 5) et les tombes en coffre de moellons sans dalles de couverture (12).

Fig. 4 - Lezha. Tombe 153 (couverture en dalles).

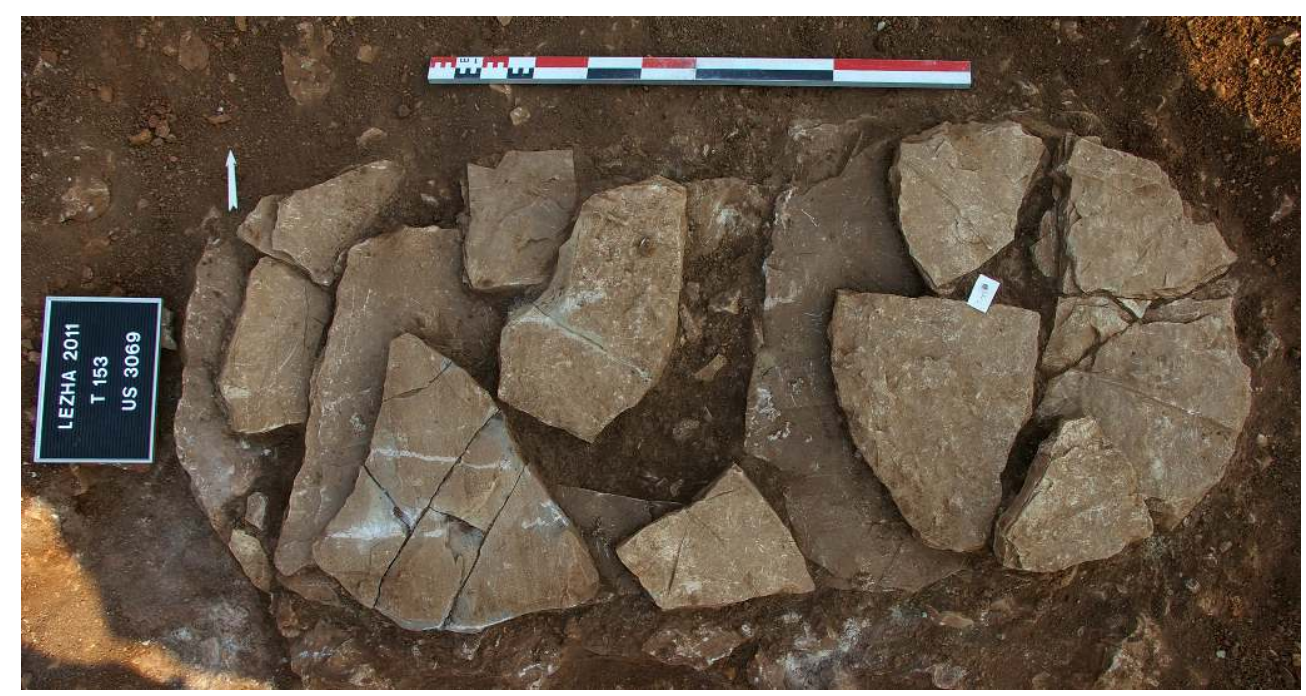


Fig. 5 - Lezha. Tombe 153.

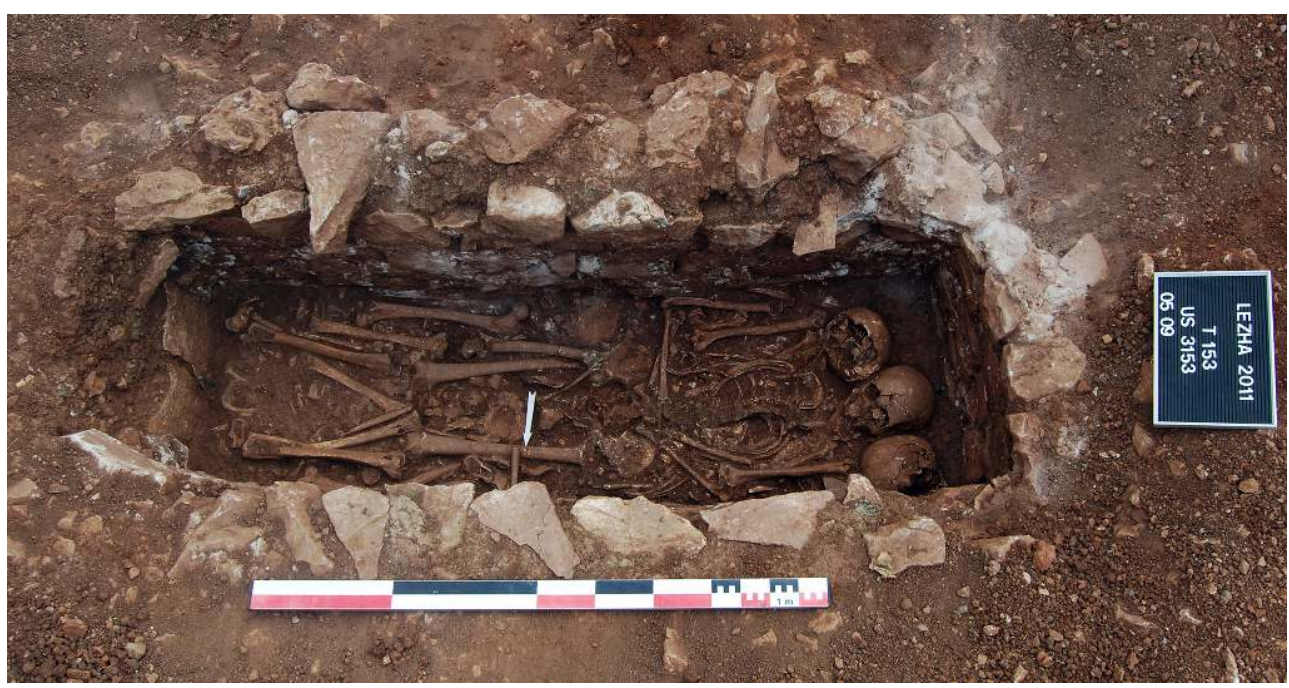

La plupart de ces tombes contiennent du mobilier funéraire, seul élément disponible actuellement pour proposer une chronologie relative des sépultures.

11 Deux autres types, déjà identifiés, se caractérisent par l'utilisation d'un cercueil de bois (attesté par de nombreux clous), qui est déposé, soit dans d'imposantes structures funéraires, à une ou plusieurs «chambres » (deux de ces tombes monumentales ont été fouillées cette année), soit dans des tombes rupestres, de grande taille et très profondes, où le cercueil était recouvert de terre et de pierres (5).

12 Certains cas se distinguent des types déjà décrits. Dans la construction de la tombe 126, très soignée, ont été utilisées, en complément des dalles et des moellons, des briques dont certaines portent les marques du briquetier (fig. 6 et 7).

Fig. 6 - Lezha. Tombe 126 (tombe mixte, dalles, moellons, briques).

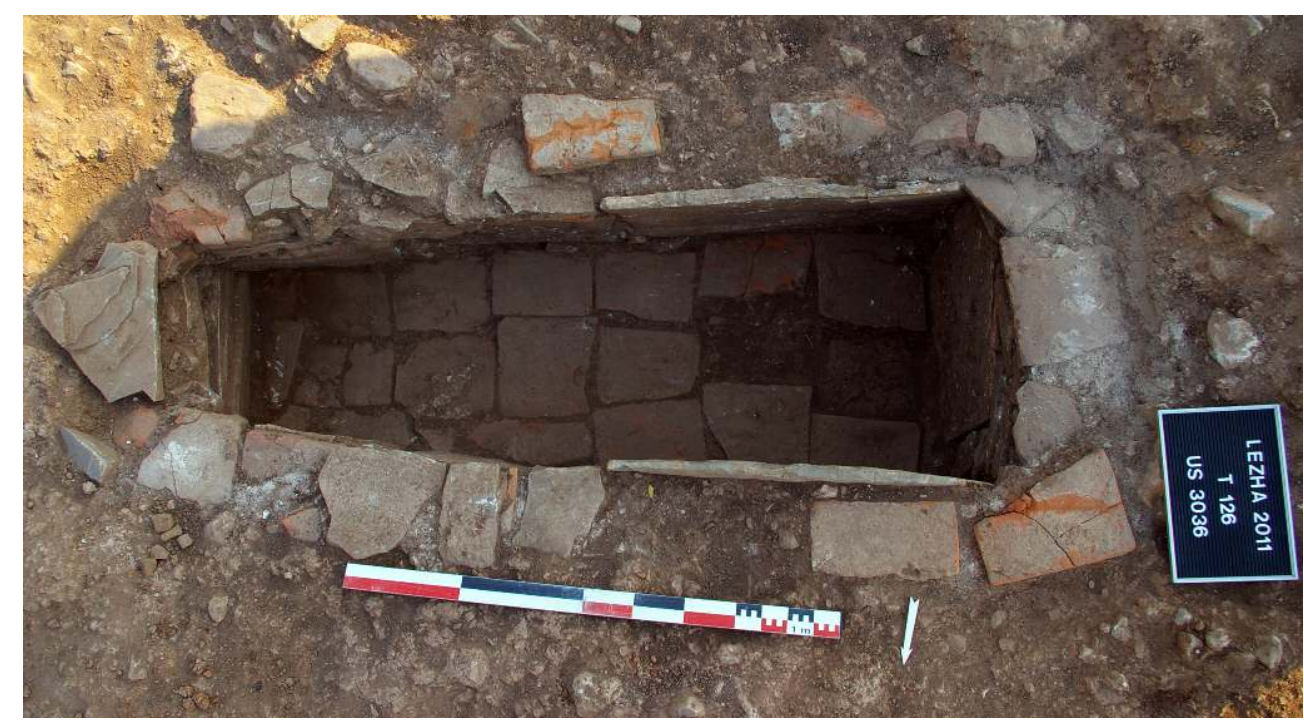


Fig. 7 - Lezha. Marque de briquetier, tombe 126.

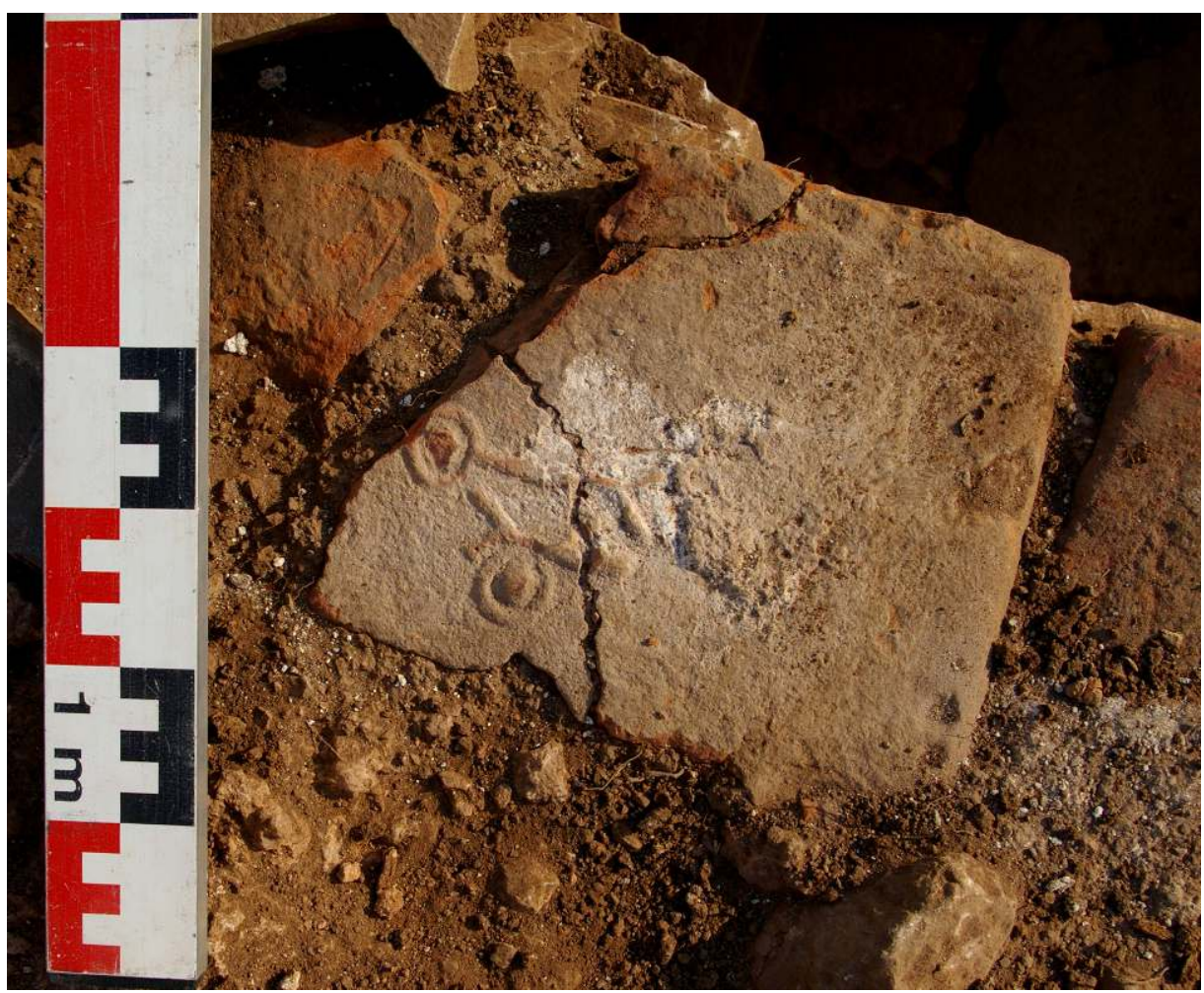

Le fond de la tombe est également dallé de briques. L'utilisation de ce matériau est rare dans l'ensemble du cimetière; on le retrouve toutefois dans les tombes 99, 134 et 137, utilisé en complément des pierres pour les parois, et dans la tombe 149 comme dallage. Notons à ce propos que l'existence d'un dallage de fond de tombe est rare, il peut être fait de briques (T. 126 et 149) ou de dalles (T. 109, 139, 146, 174). Une tombe était entièrement couverte de tuiles (T. 176), matériau que l'on retrouve en complément dans les parois des tombes 126,146 et 159 .

Enfin, deux tombes ont été creusées dans des contextes particuliers: une tombe avec cercueil de bois (T. 132) a été creusée au milieu d'une importante poche de mortier, dont l'aspect ressemble au mortier de la citerne (une étude des mortiers est programmée), et une autre (T. 178) a été creusée au fond de la citerne (cf. infra).

\section{Éléments de chronologie relative}

15 Les découvertes de 2011 confirment l'hypothèse d'une utilisation intensive de la zone située au pied de la citadelle, à l'est du monument, comme espace funéraire dès le très haut Moyen Âge. Les tombes, qui sont majoritairement faites d'un coffre de dalles, ont livré un abondant mobilier typique des $\mathrm{VI}^{\mathrm{e}}-\mathrm{VIII}{ }^{\mathrm{e}}$ siècles (fig. 8). 
Fig. 8 - Lezha. Boucle d'oreille en argent (VIe-VII siècle), tombe 177.

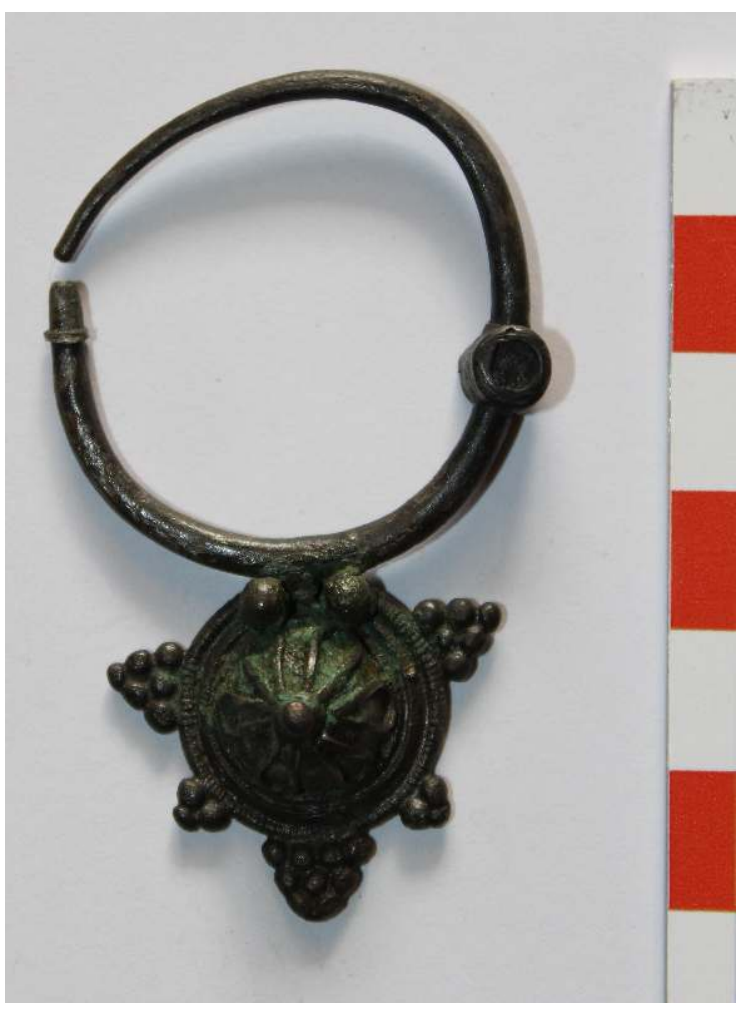

16 L'existence d'un cimetière plus ancien ne peut être exclue puisque deux crémations ont été trouvées un peu plus à l'est (fouilles de 2009). L'architecture de la tombe 126 évoque également les tombes antiques, par l'utilisation de la brique dans sa construction, mais le mobilier qui y a été retrouvé rattache l'inhumation aux premiers siècles du Moyen Âge. Toutefois, ce matériel n'a pas été retrouvé en place, car la tombe avait été bouleversée, et on ne peut être certain que le dépôt des corps a été contemporain de la construction; la tombe a très bien pu être réutilisée. Il en va peut-être de même pour la tombe 149. Sans nouvelles découvertes, ces sépultures antiques peuvent apparaître comme de simples cas isolés.

Les tombes à coffres de moellons, tous types confondus, ont livré un matériel plus récent et moins abondant (croix, bagues à motifs cruciformes...) qui les situerait entre la fin du $\mathrm{VIII}^{\mathrm{e}}$ et la fin du XII ${ }^{\mathrm{e}}$ siècle (fig. 9). Elles se répartissent majoritairement à l'est des tombes en coffre de dalles et sont plus proches des édifices religieux. 
Fig. 9 - Lezha. Pendentif cruciforme en bronze, tombe 83.

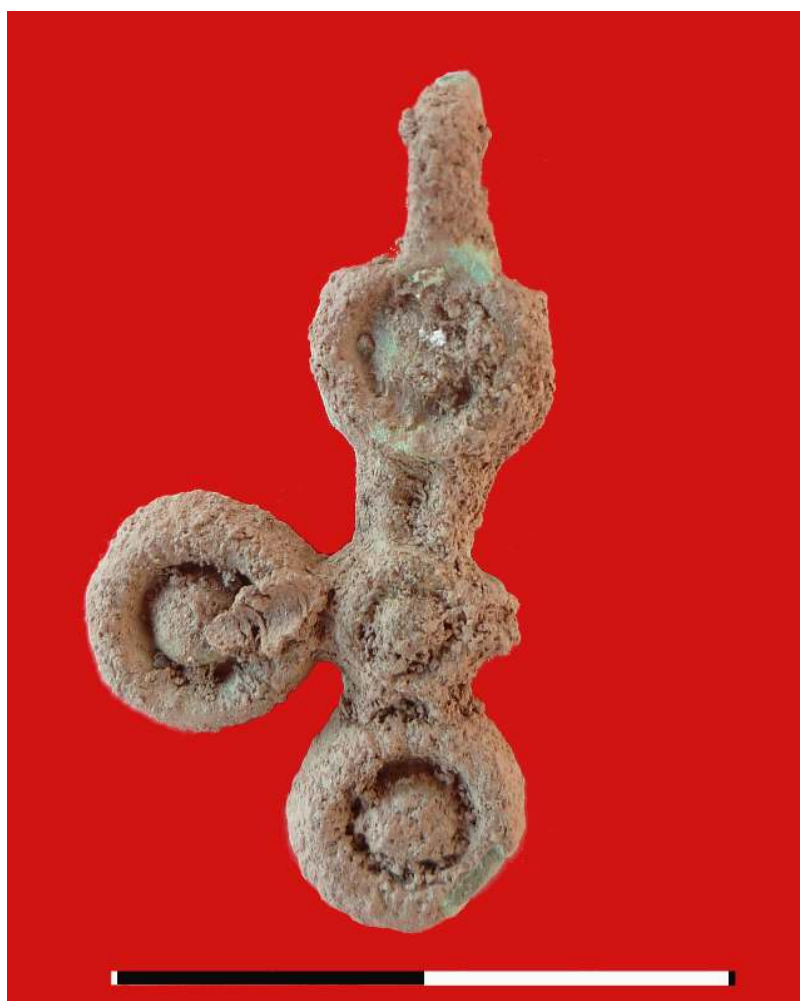

18 Les enclos et les tombes rupestres à cercueil de bois, qui ne contiennent aucun mobilier funéraire (mais qui ont livré de nombreux clous), apparaissent comme les structures funéraires les plus récentes. Les recoupements observés, notamment en zone 7, permettraient de les placer après l'abandon des édifices religieux, sans que l'on puisse, avec les éléments actuels, donner plus de précision.

\section{La chapelle (zone 7)}

$19 \mathrm{Au}$ point le plus élevé de la zone 7 , à une quinzaine de mètres au sud-est de l'église fouillée lors des précédentes campagnes, ont été mis au jour les vestiges d'une chapelle (fig. 10 et 11). 
Fig. 10 - Lezha. La chapelle, vue générale prise de l'est.

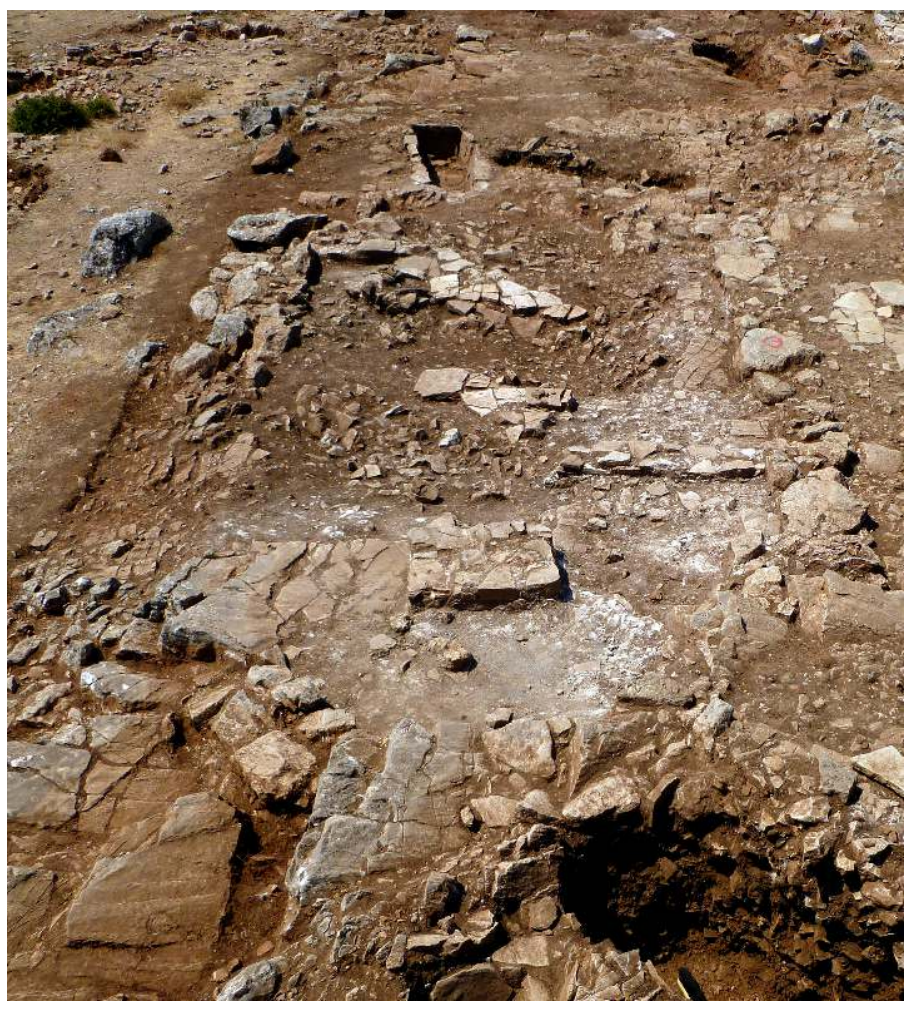

Fig. 11 - Lezha. Plan de la chapelle.

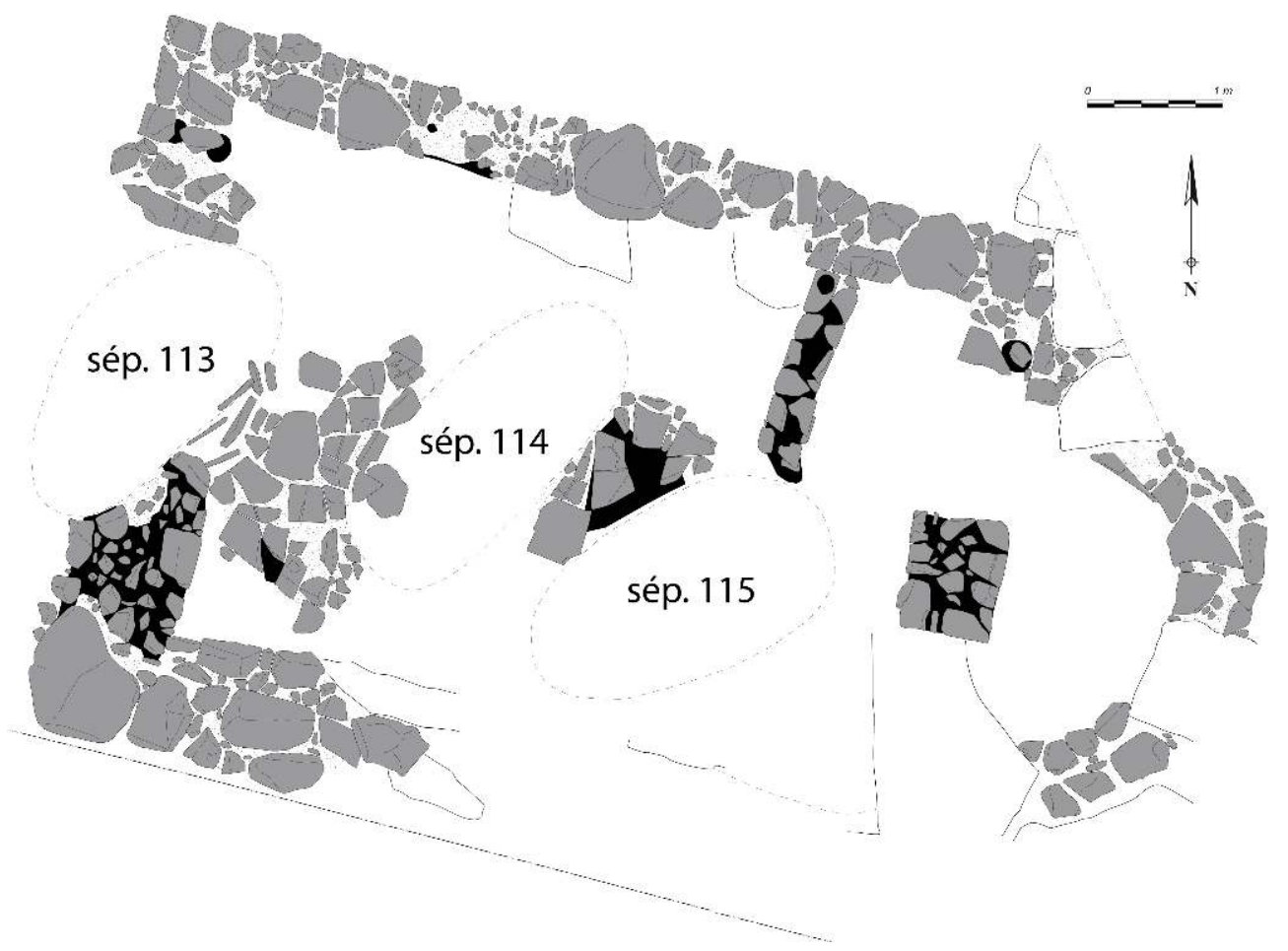

De dimensions réduites ( $7 \mathrm{~m}$ de long sur $4 \mathrm{~m}$ de large), elle est à nef unique et abside en demi-cercle. La base maçonnée de l'autel (pierres et mortier de chaux) est bien conservée ainsi que la moitié nord du stylobate de chancel. Elle est bâtie directement sur la roche 
naturelle dont certains blocs sont intégrés à la construction en association avec des pierres de tailles très variées, le tout étant lié au mortier.

21 La chapelle n'a pas encore fait l'objet de fouilles, cette zone n'étant plus menacée par la construction de la route grâce à un accord passé avec l'entreprise chargée des travaux, avec le soutien du Service albanais d'archéologie préventive. Seule la fine couche de terre végétale qui la recouvrait a été enlevée pour faire apparaître son plan et en faire un relevé topographique précis. Malgré le mauvais état de conservation des murs, le plan d'ensemble apparaît très clairement. L'intérieur de l'édifice a été intégré à l'espace funéraire après son abandon. Trois grandes tombes rupestres ont été creusées et ont détruit à cette occasion le dallage dont il ne reste que quelques éléments.

La datation de la construction, ainsi que la date de son abandon, sont encore inconnues. Seule la fouille du bâtiment et celle des tombes (que ce soit les sépultures contemporaines ou celles qui y ont été creusées après son abandon) permettra de recueillir les éléments chronologiques qui sont inconnus actuellement.

\section{La citerne (zone 1)}

Dans les derniers jours de la campagne 2009, à l'extérieur de l'angle sud-ouest de l'église, avait été dégagé un escalier de huit marches donnant accès à une vaste cuve dont apparaissait la paroi ouest, enduite de mortier de couleur orangée. La destination de cet ensemble n'avait pas été établie avec certitude et la présence d'un baptistère avait été évoquée.

L'escalier aboutit à une dalle en pierre moulurée qui isole les dernières marches de la cuve elle-même. Les parois de l'escalier sont enduites d'un mortier à la chaux ordinaire alors que celui utilisé pour la cuve elle-même évoque, par sa couleur, un mortier de tuileau, semblable à celui qui est utilisé le plus souvent pour étanchéifier une construction. Une poche de mortier de même aspect a été dégagée au nord de la chapelle. Une tombe (T.132) a été creusée en son milieu pour recevoir un cercueil de bois. Une analyse pétroarchéologique permettra de vérifier qu'il s'agit bien du même mortier.

La citerne est de forme rectangulaire. Les dimensions de la cuve ne sont pas précises en raison de destructions importantes des parois. Sa longueur est-ouest devait être légèrement supérieure à 2 mètres et sa largeur nord-sud un peu inférieure à 2 mètres. Une margelle inclinée, faite de dalles de pierre plates, dont quelques éléments subsistent au sud et au nord de la cuve, et qui pouvait délimiter le niveau supérieur de l'eau, est située à $1 \mathrm{~m}$ du fond de la cuve. Sa paroi nord est accolée aux fondations du mur sud de l'église. Les parois, conservées sur une hauteur maximum de $1,5 \mathrm{~m}$ sont faites de moellons liés au mortier et recouvertes d'une couche d'enduit hydrofuge conservé en plusieurs endroits. Le fond est plat et les angles sont arrondis (fig. 12). 
Fig. 12 - Lezha. La citerne, plan d'ensemble.

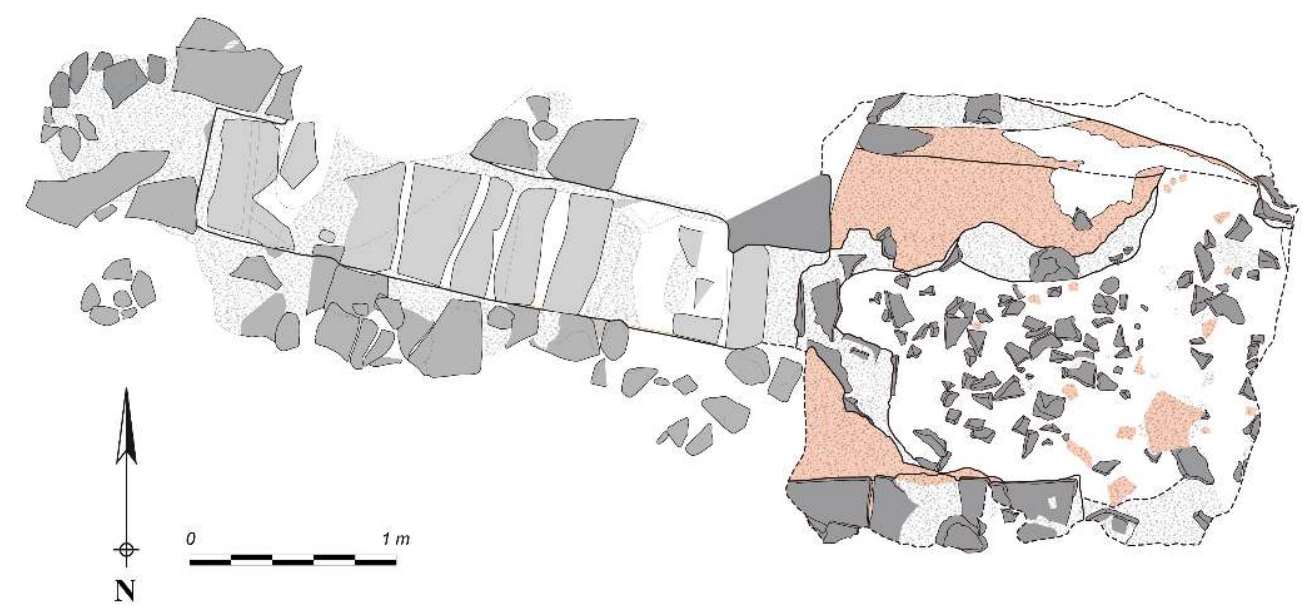

Aucun élément ne permet de savoir si elle était couverte ou à ciel ouvert, ni comment elle était approvisionnée en eau. De même, la fonction de cette construction et l'usage qui était fait de cette eau ne sont pas encore définis (simple réserve? associée à un baptistère ?). Sa proximité avec l'église évoque plutôt une utilisation associée à un rituel religieux.

Lors du dégagement de la cuve, en 2009 et en 2011, des éléments de chancel ont été retrouvés, mêlés à la terre du comblement; l'abandon de la citerne serait donc contemporain ou de peu postérieur à celui de l'église. Après son abandon, une tombe sommaire a été creusée au fond de la citerne. Cette tombe a fait l'objet d'un pillage ancien; quelques fragments osseux ainsi que quelques objets, dont une sonde-curette en argent, ont été retrouvés sur le fond de la cuve (fig. 13).

Fig. 13 - Lezha. Tombe 178, creusée au fond de la citerne.

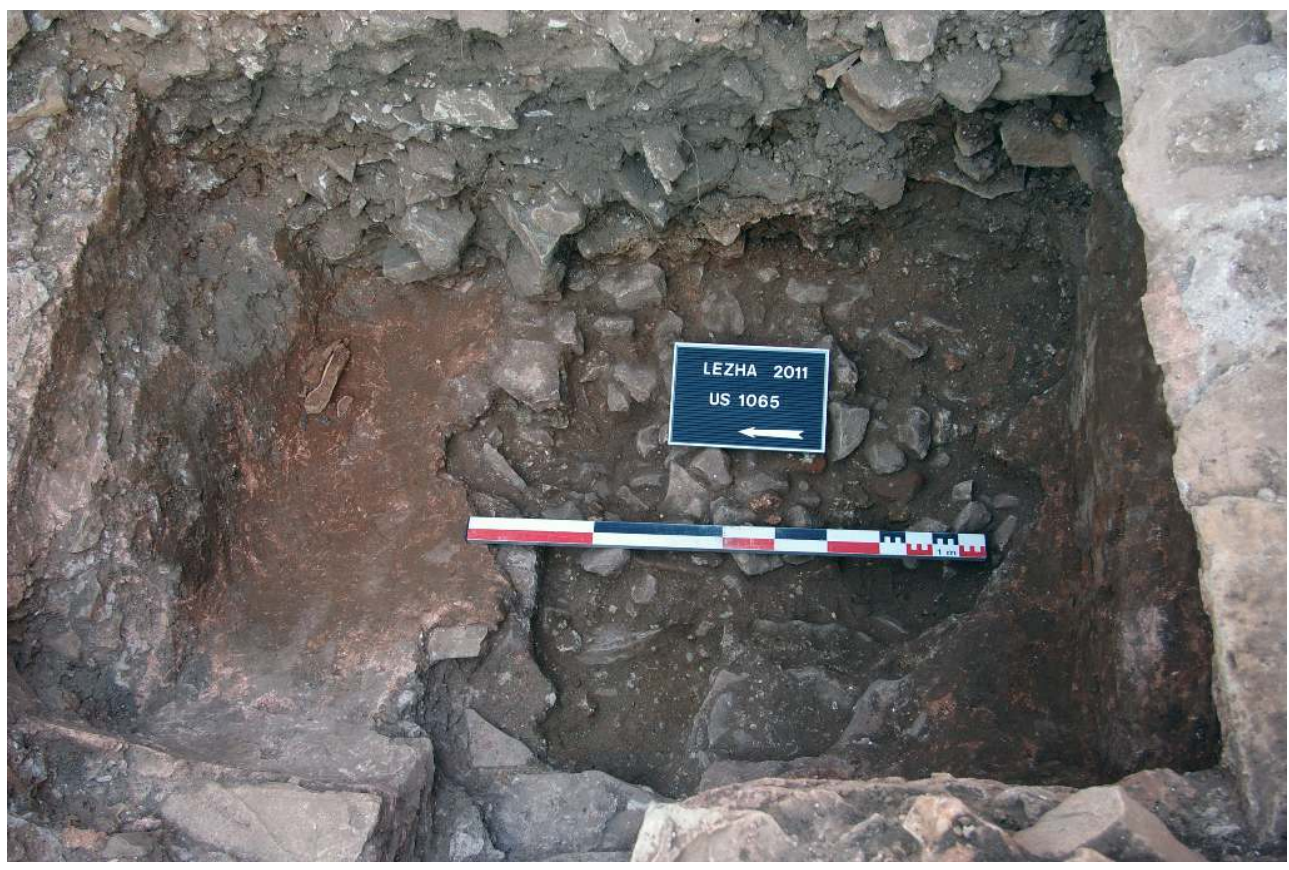


28 En 2010, cette structure a été endommagée par un pillage qui a détruit l'angle sud-est (parois et fond) de la cuve.

\section{Plan du site}

Le plan général du site a été complété par les nouvelles découvertes² (fig. 14).

Fig. 14 - Lezha. Plan général des fouilles.

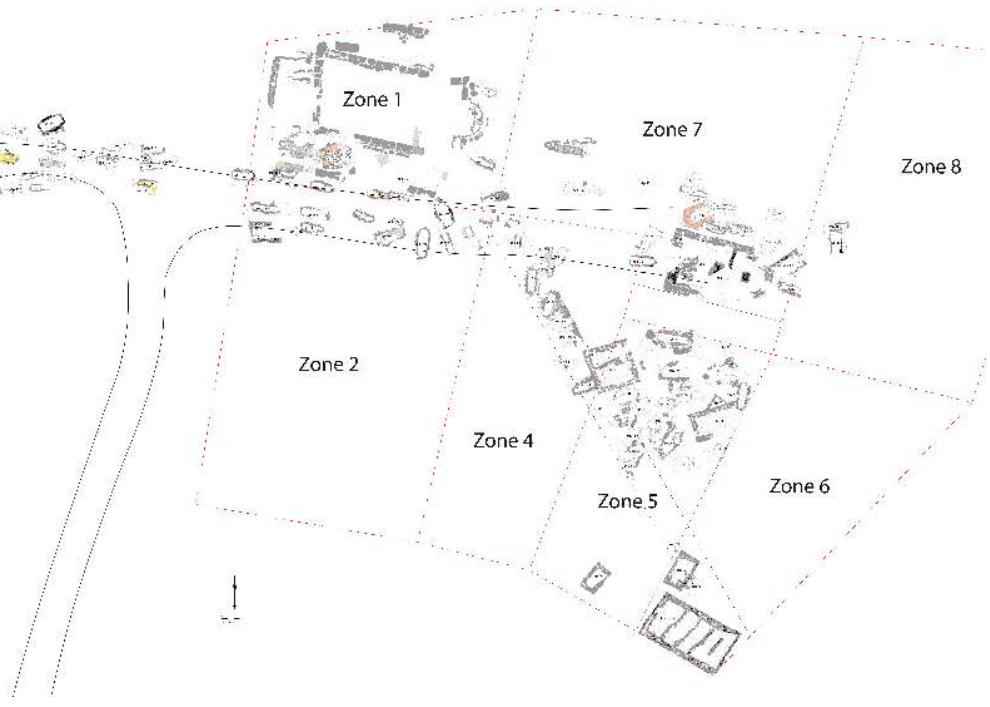

\section{Objectifs pour 2012}

Compte tenu de l'abondance de matériel recueilli en 2011, nous n'envisageons pas d'opérations de terrain en 2012, à l'exception probablement de la chapelle. En revanche, une importante campagne d'étude sera consacrée à la poursuite de l'analyse anthropologique ainsi qu'à celle de l'ensemble du mobilier mis au jour.

\section{NOTES}

1. Nous tenons à remercier spécialement: S. Gioanni, directeur des Études médiévales de l'École française de Rome et R. Chaffort, Attaché culturel de l'Ambassade de France en Albanie ; B. Beci, directeur de l'Albanian Development Fund et E. Qiriaqi de la même institution; R. Olli, directeur de l'AshA, ainsi que Sh. Gjongecaj, directrice de l'Institut archéologique d'Albanie et A. Marashi, directeur du Centre des Études albanologiques, pour leur soutien et leur disponibilité.

2. DAO S. Vattéoni et B. Bregu. 
INDEX

Mots-clés : Adriatique, haut Moyen Âge, nécropole, peuplement, anthropologie, échanges institutions École française de Rome, Centre d'histoire et civilisation de Byzance (UMR 8167 - « Orient et Méditerranée » Paris), CEPAM (UMR 6130 UNS-CNRS Nice), INED (Paris), Institut archéologique d'Albanie (Centre d'études albanologiques - Tirana), Direction de l'archéologie préventive de la Communauté d'agglomération de Douaisis (DARCAD), Service d'archéologie préventive d'Albanie (Ministère du Tourisme et de la Culture), Parc archéologique de Lezha, Municipalité de Lezha

\section{AUTEURS}

LUC BUCHET

CNRS, CEPAM - UMR 6130

\section{ETLEVA NALLBANI}

CNRS, Orient et Méditerranée - UMR 8167

\section{SURJA LELA}

Service d'Archéologie préventive d'Albanie 